\title{
A TRANSISTORLESS MICROMECHANICAL HIGH VOLTAGE GENERATOR USING A DC-POWERED SELF-OSCILLATING RELAY
}

\author{
Kabir Udeshi ${ }^{1}$ and Yogesh B. Gianchandani \\ Engineering Research Center on Wireless Integrated Microsystems \\ University of Michigan, Ann Arbor, USA
}

\begin{abstract}
This paper describes a DC powered high voltage generator that neither uses transistors nor requires a clock signal for its operation. The system, that operates on the principle of an induction coil voltage converter, is assembled using a self-oscillating mechanical relay and a wire wound inductance. Two versions of this high voltage generator have been implemented. The first version, assembled to have a footprint of less than $4 \mathrm{~mm} \times 4 \mathrm{~mm}$, uses an inductor and generates peak voltages $>200 \mathrm{~V}$ from a 4 V DC supply. An alternate version uses a transformer that provides electrical isolation and has been used to generate peak voltages $>500 \mathrm{~V}$ with a peak voltage gain $>$ 300. The self oscillating relay is fabricated using low temperature UV-LIGA process, that can be appended to a CMOS process. Experimental results used to characterize system performance show that the self oscillating relay consumes not more than $15 \%$ of input power. Circuit simulations closely track observed experimental trends.
\end{abstract}

\section{INTRODUCTION}

A large number of micromachined devices ranging from electrostatic actuators [1], to microfluidic electrokinetic pumps [2], to microplasmas [3], all need high voltages for their operation. This requirement for high voltages is what prevents the use of these devices in integrated microsytems and portable devices as they can only be powered by a single low-voltage DC supply. The need for high voltage generators extends to automotive transducers and a variety of portable electronic devices that use LCD display technology. In order to meet this demand, schemes have been proposed to integrate an additional miniaturized high voltage power source $[4,5]$. These however occupy large footprints and entail the additional overhead of power management and distribution. The preferred solution is to step up voltage from the low voltage DC power source such as a battery. A typical implementation of such a high voltage generator utilizes an inductor in conjunction with a switch that periodically breaks the current through the inductor. The miniaturization and integration of this high voltage generator has been limited by the need for an efficient inductive element as well as a high power, high voltage switch.

Traditionally microelectronic transistors have been used as switches for high voltage generators. However, in standard CMOS technology transistors are severely limited by their dielectric and junction breakdown voltages, making them unsuitable for any application that demands even moderately high voltage or power levels [6]. The use of hybrid high voltage CMOS technology results in increased costs, and yet can handle only voltages up to about $80 \mathrm{~V}$. In addition to the voltage limitation, transistors need electrical control signals that determine their switching characteristics. These control signals are generally obtained from an oscillator. The inductive element may be microfabricated, but typically results in modest inductance values [7]. An alternative approach is to integrate wire wound inductors using microassembly techniques [8].

This paper reports a high voltage generator that eliminates the need for a transistor. It operates using a fully mechanical self-oscillating relay and an assembled wire wound inductive element, without the need for any electronic circuitry. The system operates from a single DC power source. The first section describes the operation of the high voltage generator that has been implemented in two configurations. The next section reports experimental results that show a microsystem generating peak voltages in excess of $200 \mathrm{~V}$. Then experimental results are compared to with circuit simulations and further conclusions drawn.

\section{SYSTEM OPERATION}

The high voltage generator works on the principle of an induction coil voltage converter [9]. One version of the microsystem, Version I, is similar to a boost converter [10] (Fig. 1). The current through an inductor is cut periodically by the action of a switch. In the portion of the operating cycle that the switch is closed, the input current, Iin, is on, and energy is stored in the inductor. Once the current is turned off by the switch, the stored magnetic energy is converted into electric potential, which results in the generation of a high output voltage spike, Vout, across the load impedance, Zload. The cycling of the switch between its open and closed positions results in these high voltage spikes being generated at regular intervals. The pulsed

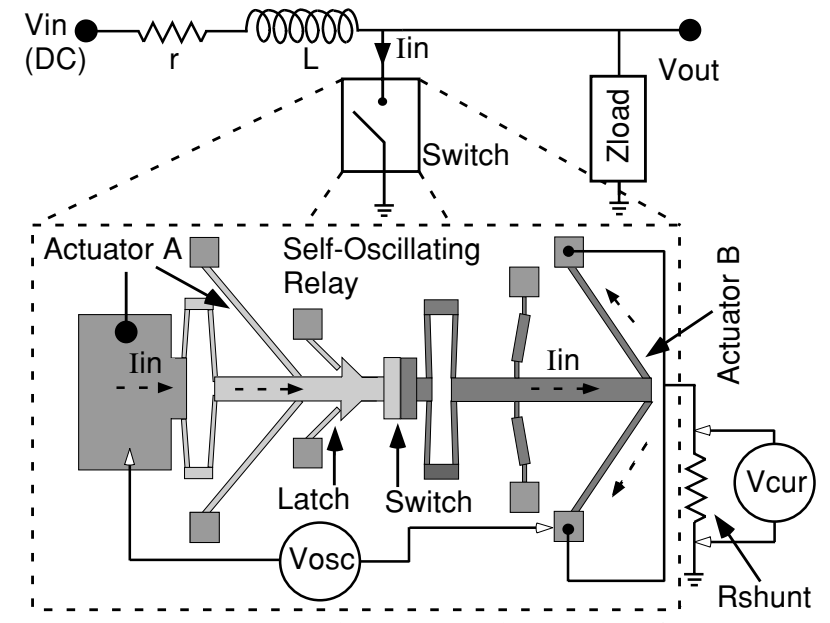

Fig. 1: Circuit diagram of Version I of the high voltage generator with a schematic of the self-oscillating relay. Rshunt $=1 \Omega$.

${ }^{1}$ Address: 1301 Beal Ave., Ann Arbor, MI 48109-2122, USA; E-mail: kudeshi@umich.edu 


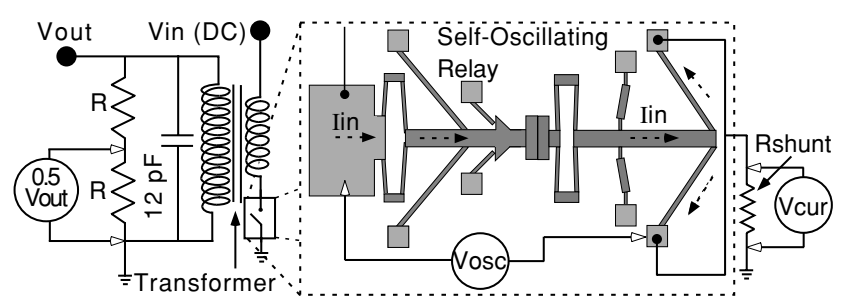

Fig. 2: Version II: High voltages have been generated using transformers that provide electrical isolation.

output could directly power a variety of devices or be converted to a high voltage DC. The periodic switching in the circuit is achieved by using a self oscillating relay [11]. The relay, operated by an electrothermal actuator, is connected directly in the current path of the circuit, and requires no additional electrical connections.

An alternative implementation, Version II, uses a miniature transformer in a manner similar to a flyback converter [12] (Fig. 2). The use of a transformer instead of an inductive coil provides electrical isolation as well allows for the capability to generate higher output voltages by choosing a suitable turns-ratio.

\section{CIRCUIT ANALYSIS}

Both versions of the high voltage generator may be analyzed using the circuit in Fig. 1 consisting of a DC voltage source, Vin, parasitic resistance of the coil winding, $r$, an inductor, $L$, a switch, and the load impedance, Zload. The circuit has two phases of operation. In the first phase, the switch is closed and the current through the circuit ramps up resulting in energy being stored in the inductor. The differential equation that describes the change in current, Iin, with time, $t$, during this phase of operation and its solution is given by [9]:

$$
r \operatorname{Iin}+L \frac{d \operatorname{Iin}}{d t}=\operatorname{Vin} \quad ; \quad \operatorname{Iin}=\frac{\operatorname{Vin}}{r}\left(1-e^{\frac{-r t}{L}}\right)
$$

The equation is derived under the assumption that when the switch is closed, no current flows through Zload, which is much larger than the parasitic resistance of the closed switch.

The circuit goes into its second phase of operation at the instant the switch starts opening. As the current falls to zero, the e.m.f. of the coil adds itself to that of the source leading to a high voltage across the output load. Since there are numerous physical phenomena occurring at this moment, including potential arcing across the switch, it is not easily described by a closed form expression. However, the output voltage generated, Vout, is known to be a function of the rate of change of current, Iout, that flows through the load during this phase of operation [9].

Version II of the high voltage generator may be

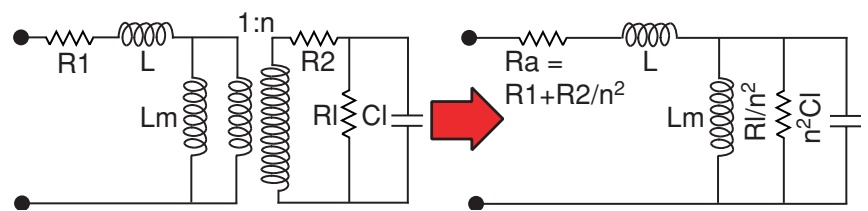

Fig. 3: The model of a transformer can be converted to an equivalent circuit with an inductor.

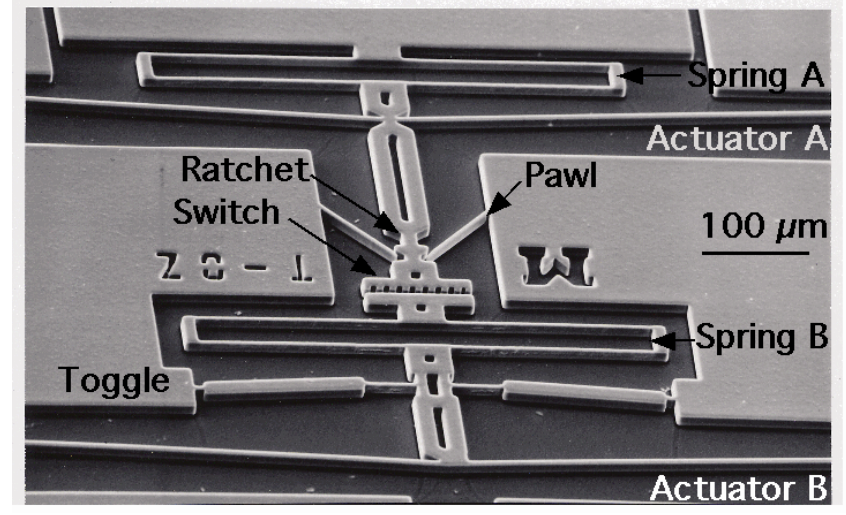

Fig. 4: SEM image of fabricated self-oscillating relay.

analyzed in a similar manner by converting the model of a transformer into an equivalent circuit containing inductors. Figure 3 shows the model of a transformer where $R 1, R 2$ represent the primary and secondary coil resistances respectively, $L$ the leakage inductance, $L m$ the magnetization inductance, $n$ the turns ratio and $R l$ and $C l$ represent the load resistance and capacitance, respectively [13]. Lm corresponds to the inductance that produces magnetic flux in the transformer. The equivalent circuit eliminates the transformer and scales the load connected to the secondary circuit by a factor determined by the turns ratio. This circuit can be simulated using SPICE. The parameter that needs to be set is the rate of change of output current, which can be derived from experimental data.

\section{FABRICATION \& ASSEMBLY}

The self-oscillating switch was fabricated using a single mask UV-LIGA process using SU-8 resist [14]. Thirty micron thick structures were fabricated from electroplated copper with a minimum feature size of $3 \mu \mathrm{m}$. A SEM of the oscillator is shown in Fig. 4. The low temperature process used to fabricate the switch allows it to be appended to a CMOS process.

The coil was wound using a coil winder on a cylindrical steel core, and assembled onto a PCB along with the die of the oscillator. The microsystem shown in Fig. 5 has a footprint of $4 \mathrm{~mm} \times 4 \mathrm{~mm}$. The dimensions of the inductor may be significantly reduced by the use of toroidal, nickeliron cores.

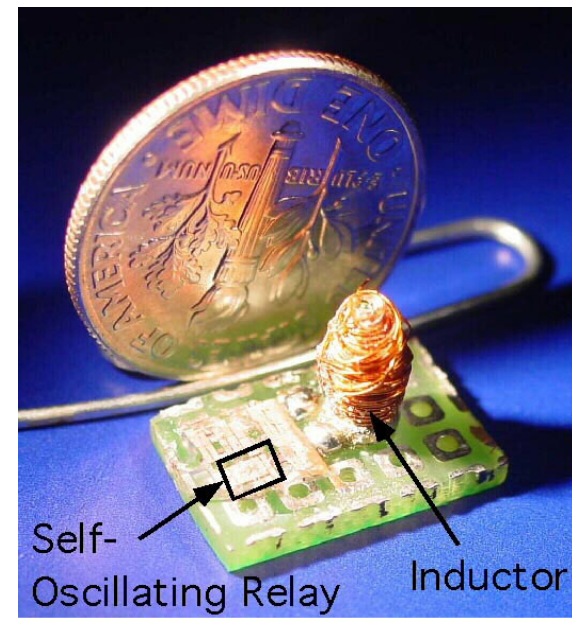

Fig. 5: A

photograph of Version I of the high voltage generator made by assembling the self-oscillating relay with a wire wound inductor 

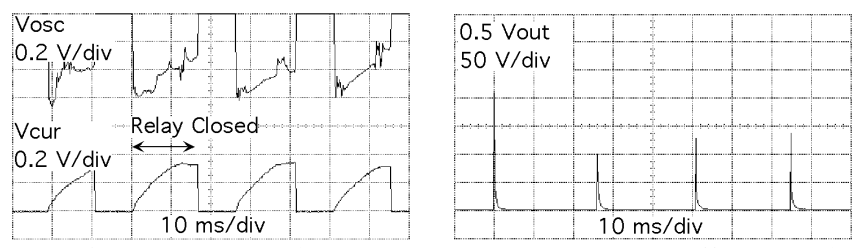

Fig. 6: Output waveforms as obtained on an oscilloscope for Version II of the high voltage generator.

\section{MEASUREMENT RESULTS}

The system was tested and characterized for a variety of input parameters and operating conditions. The experimental characteristics were compared with circuit simulations to explain trends observed.

To verify the operation of the high voltage generator, the nodal voltages and circuit current were dynamically recorded using the setup of Fig 2. When the switch (Fig. 2) is closed, the current flowing through the primary of the transformer, Vcur, ramps up (Fig. 6), and the voltage across the relay, Vosc, is at a small value corresponding to the small resistive voltage drop across a closed switch. Opening of the switch causes Vosc to rise to the supply voltage and the current to drop rapidly leading to the generation of a high voltage, Vout, across the load. Variations in the magnitude of peak Vout could be due to digital sampling carried out by the oscilloscope. The high voltage spikes do not temporally coincide with the location of the falling current because separate oscilloscopes were used to capture these waveforms using independent trigger signals. The observed waveforms verify the operation of the high voltage generator as described by the theory of an induction coil voltage converter.

In Version I, the self-oscillating relay has been integrated with a hand wound $450 \mu \mathrm{H}$ inductance, as shown in Fig. 5, and has been used to generate voltages up to 208 $\mathrm{V}$ from a $3.8 \mathrm{~V}$ DC supply while driving a $1 \mathrm{M} \Omega, 12 \mathrm{pF}$ load. The peak output voltage (Fig. 7) increases with input power (a), while the peak voltage gain remains constant at about 50 (b). In power calculations, it is assumed that while the switch is closed, no current flows through the output load. This assumption is valid as the resistance of the relay in the closed state $(\approx 15 \Omega)$ is negligible compared to that of the output load (which is in the $\mathrm{K} \Omega$ to $\mathrm{M} \Omega$ range). Thus, Vosc $\mathrm{x}$ Iin gives the input power consumption by the relay, while Vin $x$ Iin gives the total input power. The fraction of input power consumed by the self-oscillating relay is 5-14 $\%$ (Fig. 7c). Since up to $95 \%$ of the input power is delivered to the inductor at high output voltage levels, optimized circuit parameters can result in acceptable energy efficiency.

Simulated values of peak output voltage obtained, track experimental results (Fig 7a). In order to simulate circuit performance, an input parameter required is the rate of change of current. The self-oscillating relay was modeled as a linear resistor whose resistance changes from $0 \Omega$ to $100 \mathrm{M} \Omega$ as the switch opens. The time rate of change of this resistance is used to determine the rate of change of current. A resistance change time of $450 \mu$ s allows simulated results to track those obtained experimentally.
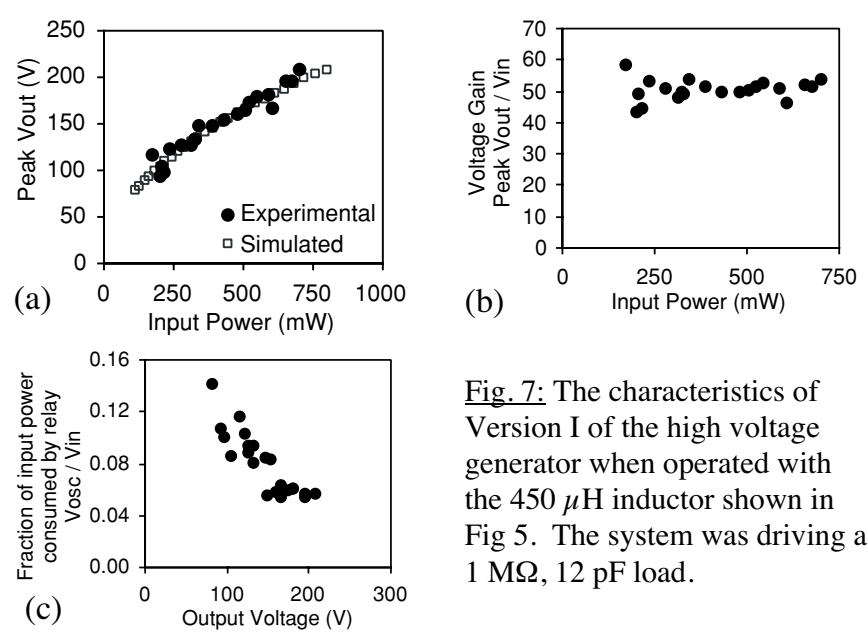

Fig. 7: The characteristics of Version I of the high voltage generator when operated with the $450 \mu \mathrm{H}$ inductor shown in Fig 5. The system was driving a $1 \mathrm{M} \Omega, 12 \mathrm{pF}$ load.

Version II of the high voltage generator uses a transformer and provides electrical isolation between the input and output as shown in Fig. 2. A study of the operating characteristics under different resistive loading conditions was carried out, using a transformer with a magnetization inductance of $3.5 \mu \mathrm{H}$ and $12 \mathrm{pF}$ capacitive load in parallel with the resistive load (Fig. 8). The peak output voltage (a) and voltage gain (b) decrease with smaller load resistance values due to higher current flow rates. The higher currents also cause an increase in the input power consumed by the microsystem (c). The low fraction of input power consumption by the relay $(<15 \%)$ indicates that even though driven by an electrothermal actuator, it is energy efficient (d).

In order to simulate the circuit performance, the parameters $L m, L, n, R 1$ and $R 2$ of a physical transformer need to measured. The coil resistances $R 1$ and $R 2$ can be measured using an ohmmeter. The other parameters are calculated using [15]:

$$
\begin{aligned}
& L m=M^{2} / L_{2} O \quad ; \quad L=L_{1} o-M^{2} / L_{2} O \\
& n=L_{2} O / M \quad ; \quad M=\sqrt{L_{1} O\left(L_{2} o-L_{1} s\right)}
\end{aligned}
$$

where, $L$, is the measured inductance, with the first subscript indicating the side of the transformer; 1 indicates the primary and 2 the secondary. The second subscript indicates the condition of the adjacent winding while
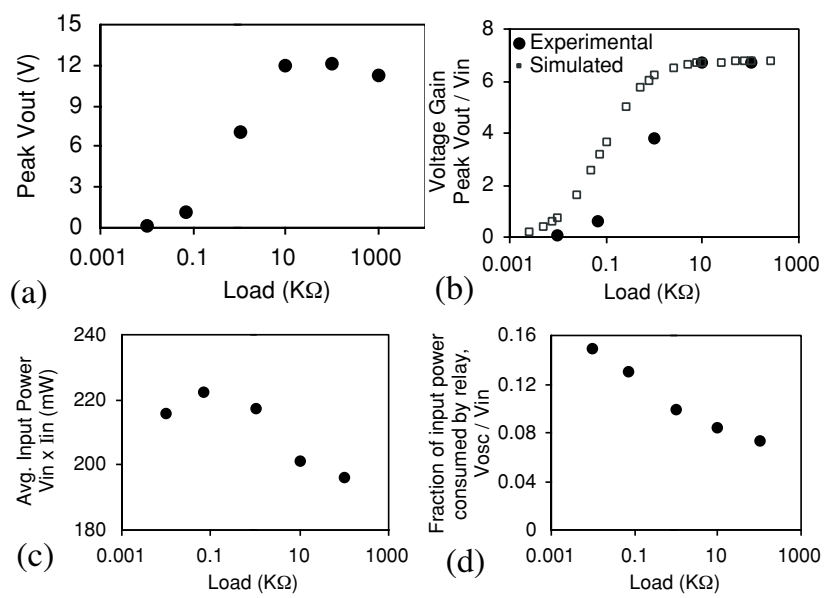

Fig. 8: Version II tests: A study of the operating characteristics under different resistive loading conditions. 


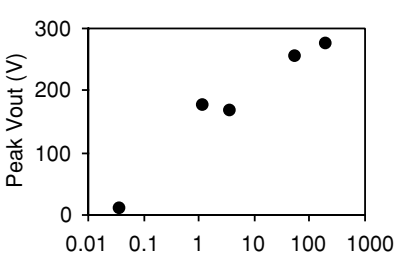

(a) Magnetization Inductance $(\mathrm{mH})$
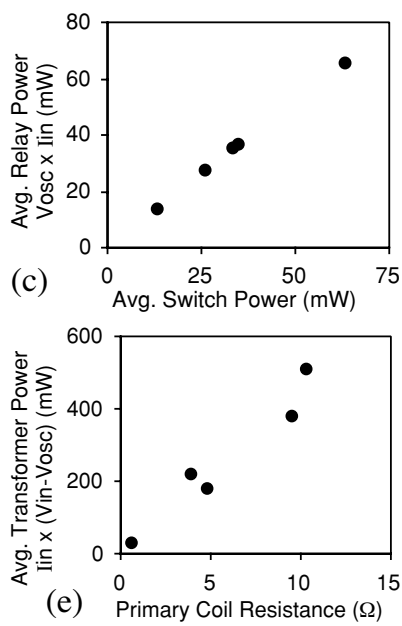

(e) Primary Coil Resistance $(\Omega)$
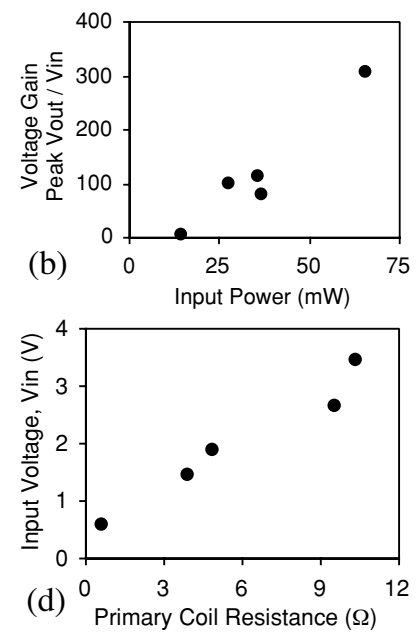

Fig. 9: Version II tests; by using transformers with different magnetization inductances, the output voltage may be controlled and has been experimentally analyzed while driving a 100 $\mathrm{K} \Omega, 12 \mathrm{pF}$ load.

carrying out the inductance measurement; whether open, $o$, or shorted, $s$.

The measured transformer characteristics were used to simulate the performance of the circuit and are compared with experimentally obtained values of peak output voltage in Fig. 8(b). The S-shaped curve can be explained by observing the nature of the load. At small values of load resistance, most of the output current flows through the resistor. However at high values of resistive load, the charging current of the capacitor becomes dominant. Since a fixed capacitance was used while carrying out the experiments, the peak voltage gain saturates at large resistive loads.

Output voltages generated by the system may be changed by varying the magnetization inductance, $L m$, of a transformer (Fig 9). The output voltage (a) and voltage gain (b) increase with higher values of inductance due to greater energy storage capacity. The input voltage of the system (d), as well as transformer power consumption (e) depends significantly on coil resistance, which may be greatly reduced by the use of superior winding coil material. Power consumption of the oscillating relay is found to depend mainly on the power drop across the contact resistance of the relay switch $(\mathrm{c})$.

\section{CONCLUSIONS}

The high voltage generator reported in this paper can generate high voltage spikes from a DC source without the use of transistors. Version I of this system uses an inductor and has been assembled along with a self oscillating relay to have a footprint of less than $4 \mathrm{~mm} \times 4 \mathrm{~mm}$ and can generate voltages in excess of $200 \mathrm{~V}$ from a $4 \mathrm{~V}$ DC supply. Version II uses a transformer that provides electrical isolation and allows greater control over the output voltage through the turns ratio. The system performance has been characterized

under various power levels, loads and inductance values. The self-oscillating relay consumes less than $15 \%$ of the input power indicating that the system has a high energy efficiency. Results of circuit simulations, with a single fitting parameter, closely track experimental results, indicating that the system operates as predicted by theory. A reliable and optimized design of the high voltage generator reported can power high voltage sensors and allow complete integrated microsystems to operate using a single a low voltage DC power source.

\section{ACKNOWLEDGEMENTS}

This work was supported primarily by the Engineering Research Centers Program of the National Science Foundation under Award Number EEC-9986866. The facilities used for this research include the Solid-State Electronics Laboratory (SSEL) at the University of Michigan. Travel support has been generously provided by the Transducers Research Foundation and by the DARPA MEMS and DARPA BioFlips programs

\section{REFERENCES}

[1] W. Tang, T. Nguyen, M. Judy, R. Howe, "Electrostatic CombDrive of Lateral Polysilicon Resonators," Sensors and Actuators A (Physical), v A21, n 1-3, Feb. 1990, p 328-31

[2] S. Zeng, H. Chen, J. Mikkelsen, J. Santiago, "Fabrication and characterization of electrokinetic micro pumps," Thermomechanical Phenomena in Electronic Systems, v 2, 2000, p 31-36

[3] C. Wilson, Y. Gianchandani, "Spectral Detection of Metal Contaminants in Water using an On-Chip Microglow Discharge," IEEE Trans. Electron Devices, v 49, n 12, Dec. 2002, p 2317-22

[4] J. Lee, Z. Chen, M. Allen, A. Rohatgi, R. Arya, "A miniaturized high-voltage solar cell array as an electrostatic MEMS power supply," J. MEMS, v 4, Sept. 1995, p102-108

[5] J. Bates, G. Gruzalski, C. Luck, "Rechargeable solid state lithium microbatteries," IEEE MEMS, 1993, p 82-86

[6] H. Jiang, W. Carr, "On-chip integration of high-voltage generator circuits for an electrostatic micromotor," Intl Conf. on Solid-State Sensors and Actuators, v 1, 1995, p 150-53

[7] C. Ahn, M. Allen, "A comparison of two micromachined inductors (bar and meander-type) for fully integrated boost DC/DC power converters," IEEE Transactions on Power Electronics, v 11, n 2 , March 1996, p 239-45

[8] G. Skidmore, M. Ellis, A. Geisberger, K. Tsui, K. Tuck, R. Saini, T. Udeshi, M. Nolan, R. Stallcup, J. Von Ehr II, “Assembly Technology Across Multiple Length Scales From the Micro- Scale to the Nano-Scale," IEEE MEMS, 2004, p 588-92

[9] H. Armagnat, The theory, design and construction of induction coils, New York, McGraw publishing company, 1908

[10] R.Jaeger, Microelectronic Circuit Design, McGraw-Hill, 1997

[11]K. Udeshi, Y. Gianchandani, "A DC-Powered, Tunable, Fully Mechanical Oscillator Using In-plane Electrothermal Actuation," IEEE MEMS, 2004, p 502-06

[12]N. Mohan, T. Undeland, W. Robbins, Power electronics: converters, applications, and design, New York, John Wiley \& Sons, Inc., 1995

[13]W. Flanagan, Handbook of transformer design and applications, McGraw-Hill, Inc., 1993

[14]H. Lorents, M. Despont, N. Fahrni, N. LaBianaca, P. Renaud, P. Vettiger, "SU-8: a low-cost negative resist for MEMS," $J$. Micromech. Microeng , v 7, n 3, 1997, p 121-24

[15] J. Stewart, A. Boyd, Theory and design of capacitor-storage ignition systems, Technical Report, Univeristy of Michigan, Ann Arbor, 1956 